Special issue of the 3rd International Conference on Computational and Experimental Science and Engineering (ICCESEN 2016)

\title{
Conformable Fractional Gradient Based Dynamic System for Constrained Optimization Problem
}

\author{
F. EvirGEN* \\ Balıkesir University, Department of Mathematics, Balıkesir, Turkey
}

\begin{abstract}
A conformable fractional gradient based dynamic system with a steepest descent direction is proposed in this paper for a class of nonlinear programming problems. The solutions of the dynamic system, modelled with the conformable fractional derivative are investigated to obtain the minimizing point of the optimization problem. For this purpose, we use a step variational iteration method, adapted to use a conformable integral definition. Numerical simulations and comparisons show that the conformable fractional gradient based dynamic system is both feasible and efficient for a certain class of equality constrained optimization problems. Furthermore, the step variational iteration method, combined with the conformable integral definition, is a reliable tool for solving a system of fractional differential equations.
\end{abstract}

DOI: 10.12693/APhysPolA.132.1066

PACS/topics: 02.60.Pn, 45.10.Hj, 31.15.Pf

\section{Introduction}

Optimization is an interdisciplinary subject, used by scientists from various disciplines, to make the best possible decisions. This ensures that optimization is always an interesting research topic for scientists [1-4]. In the literature, several efficient methods have been put forward to obtain the minimizing point of these problems. A detailed and modern discussion of these methods can be found in [5].

The gradient based method is one of these approaches, which was first introduced by Arrow and Hurwicz [6] to solve optimization problems. The main idea behind the method is to replace the optimization problem with a system of ordinary differential equations, which are equipped with optimality conditions, in order to obtain the optimal solutions to the nonlinear programming problem. Numerous works on this approach can be found in the literature [7-17].

Fractional calculus, is another important scientific research area [18], since many real-life problems can be modelled in a more stable way with fractional derivatives. Many researchers in this field have mainly focused on developing analytical and numerical methods for solving different kinds of fractional differential equations. The variational iteration method (VIM) is one of these methods and was first introduced by He [19]. Recently, Molliq et al. [20] defined the step VIM (SVIM) for obtaining the essential behaviour of the system for a large time $t$.

In this paper, we construct a conformable fractional gradient based system for solving an equality constrained optimization problem. The conformable fractional derivative has been recently defined by Khalil et al. [21].

*e-mail: fevirgen@balikesir.edu.tr
It is a natural extension of the usual derivative, and this local operator preserves many of the properties of the classical derivative (see $[21,22])$. The proposed system shows that the steady state solutions $x(t)$ of the system can be approximated to the optimal solutions $x^{*}$ of the optimization problem on a continuous path as $t \rightarrow \infty$. The step variational iteration method (SVIM) is used to achieve the intended results with a conformable derivative and integral definitions.

\section{Preliminaries}

\subsection{Equality constrained optimization problem}

Consider the nonlinear programming problem with equality constraints:

$$
\min _{x \in \mathbb{R}^{n}} f(x) \text {, s.t. } h(x)=0,
$$

where $f: \mathbb{R}^{n} \rightarrow \mathbb{R}, h: \mathbb{R}^{n} \rightarrow \mathbb{R}^{p}$ are $C^{2}$ functions. The penalty methods aim to approximate a constrained optimization problem by a sequence unconstrained optimization problem. A well-known penalty function for the problem in Eq. (1) is given by

$$
P_{\text {penalty }}(h(x))=\frac{1}{\gamma} \sum_{i=1}^{p}\left(h_{i}(x)\right)^{\gamma},
$$

where $\gamma>0$ is a constant. It can be shown that under some conditions the solutions to the problem in (1) are the solutions of the following unconstrained one:

$$
\min F(x, \eta)=f(x)+\eta \frac{1}{\gamma} \sum_{i=1}^{p}\left(h_{i}(x)\right)^{\gamma},
$$

$$
\text { s.t. } x \in \mathbb{R}^{n} \text {, }
$$

where $\eta>0$ is an auxiliary penalty variable and is sufficiently large. One of the main results connecting the minimizers of the problem in Eq. (1) and the unconstrained problem in Eq. (3) can be described as follows.

Theorem $1\left[5\right.$, p. 404] Let $\left\{x_{k}\right\}$ be a sequence generated by the penalty method. Then any limit point of the sequence is a solution to the constrained problem. 


\subsection{Conformable fractional derivative}

Fractional calculus generates great interest among scientists, and numerous studies on fractional derivatives and fractional integrals have been undertaken. The most popular definitions in the literature are the RiemannLiouville's fractional derivative and the Caputo fractional derivative. These fractional derivative definitions are generally used for the mathematical modelling within many areas, when the usual integer order derivative operator is not sufficient.

However, the greatest deficiency of these two definitions is that they do not provide some of the features that the classical derivative provides, such as the derivative of constant and the product rule. Recently Khalil et al. in [21] have characterized a new fractional derivative operator, known as the conformable fractional derivative, to overcome these deficiencies. Besides these advantages, the conformable fractional derivative does not show the memory effect, which is inherent for the other classical fractional derivatives.

Definition 1 Let $f:[0, \infty) \rightarrow \mathbb{R}$ be a function. The $\alpha^{t h}$ order conformable fractional derivative of $f$ is defined by

$$
T_{\alpha}(f)(t)=\lim _{\varepsilon \rightarrow 0} \frac{f\left(t+\varepsilon t^{1-\alpha}\right)-f(t)}{\varepsilon},
$$

for all $t>0$ and $\alpha \in(0,1)$.

This new definition preserves many properties of the classical derivative $[21,22]$. Some of the features that we will use are as follows.

Theorem 2 Let $0<\alpha \leq 1$ and $f, g$ be $\alpha$-differentiable at a point $t>0$. If $f$ is a differentiable function then $\frac{\mathrm{d}^{\alpha} f}{\mathrm{~d} t^{\alpha}}=t^{1-\alpha} \frac{\mathrm{d} f}{\mathrm{~d} t}$.

Definition $3 I_{\alpha}^{a}(f)(t)=I_{1}^{a}\left(t^{\alpha-1} f\right)=\int_{a}^{t} \frac{f(x)}{x^{1-\alpha}} \mathrm{d} x$, where the integral is the usual Riemann improper integral, and $\alpha \in(0,1)$.

Theorem 3 Let $f$ be any continuous function in the domain of $I_{\alpha}$. Then $T_{\alpha} I_{\alpha}^{a}(f)(t)=f(t)$, for all $t \geq a$.

Theorem 4 Let $f:(a, b) \rightarrow \mathbb{R}$ be differentiable and $0<\alpha \leq 1$. Then for all $t>a$ we have $I_{\alpha}^{a} T_{\alpha}(f)(t)=$ $f(t)-f(a)$.

\subsection{Variational iteration method}

Let us consider the following general nonlinear differential equation to express the VIM,

$$
L(u(t))+N(u(t))=g(t),
$$

where $L$ is a linear operator, $N$ is a nonlinear operator and $g(t)$ is a known analytical function [19]. We can construct a correction functional for (4) as follows,

$$
\begin{aligned}
& u_{i, k+1}(t)=u_{i, k}(t) \\
& +\int_{t_{0}}^{t} \lambda(\tau)\left\{L\left(u_{i, k}(\tau)\right)+N\left(\tilde{u}_{i, k}(\tau)\right)-g(\tau)\right\} \mathrm{d} \tau,
\end{aligned}
$$

where $\lambda$ is a general Lagrange multiplier, which can be identified optimally via variational theory, and $k$ is the $k$ th approximation. Here $\tilde{u}_{i, k}$ is a restricted variation and $\delta \tilde{u}_{i, k}=0$. Finally, the exact solution may be obtained as

$$
u_{i}(t)=\lim _{k \rightarrow \infty} u_{i, k}(t) .
$$

\section{Conformable fractional gradient based dynamic system}

Consider the optimization problem with equality constraints defined by Eq. (1). Generally, these types of problems are solved by transforming to the unconstrained optimization problem (3). In the next step, some traditional methods are used to minimize the unconstrained problem.

In this article, a conformable fractional gradient based dynamical system is handled for obtaining optimal solutions of (1) with the help of the step variational iteration method. The conformable fractional derivative is used for modelling. This kind of fractional gradient based approach for solving optimization problems was first introduced by Evirgen and Özdemir [23-25].

Utilizing the penalty function (2) for problem (1) with $\gamma=2$, the conformable fractional gradient based dynamic model can be defined by the following expression:

$$
\begin{array}{ll}
T_{\alpha} x(t)=-\nabla_{x} F(x, \eta), & 0<\alpha \leq 1, \\
x_{i}(0)=x_{i 0}, & i=1 \ldots n,
\end{array}
$$

where $\nabla_{x} F(x, \eta)$ is the gradient vector of (2) with respect to the $x \in \mathbb{R}^{n}$.

Definition 4 A point $x_{e}$ is referred to as an equilibrium point of (6) if it satisfies the right-hand side of Eq. (6).

The conformable fractional gradient based dynamic system (6) can be expressed in a more convenient form as follows:

$$
T_{\alpha} x_{i}(t)=g_{i}\left(t, \eta, x_{1}, x_{2}, \ldots, x_{n}\right), i=1,2, \ldots, n .
$$

The stable equilibrium point of the conformable fractional order system (7) is acquired with the SVIM algorithm. The SVIM can be described in terms of certain modifications to VIM. In this approach, to ensure the validity of the approximations of VIM for large $t$, we need to treat (5) under the equally divided intervals of $\left[t_{0}, t\right)$ such as $\left[t_{0}, t_{1}\right),\left[t_{1}, t_{2}\right), \ldots,\left[t_{j-1}, t_{j}=t\right)$.

The correction functional for the system of conformable fractional nonlinear differential equation (7), according to the SVIM, can be constructed as

$$
\begin{gathered}
x_{i}^{k+1}(t)=x_{i}^{k}(t)+I_{\alpha}^{t^{*}}\left(\lambda _ { i } ( \tau ) \left(T_{\alpha} x_{i}^{k}(\tau)\right.\right. \\
\left.\left.-g_{i}\left(\tilde{x}_{1}^{k}(\tau), \ldots, \tilde{x}_{n}^{k}(\tau)\right)\right)\right) \mathrm{d} \tau,
\end{gathered}
$$

where $t^{*}$ is the left end point of each subinterval, $\lambda_{i}$, $i=1,2, \ldots, n$ are general Lagrange multipliers, which can be identified optimally via variational theory, and $\tilde{x}_{1}$, $\tilde{x}_{2}, \ldots, \tilde{x}_{n}$ denote restricted variations, such that $\delta \tilde{x}_{i}=0$.

By taking variation with respect to the independent variable $x_{i}, i=1,2, \ldots, n$, with $\delta x_{i}\left(t^{*}\right)=0$,

$$
\begin{aligned}
& \delta x_{i}^{k+1}(t)=\delta x_{i}^{k}(t)+\delta \int_{0}^{t-t^{*}} \lambda_{i}(\tau)\left(x_{i}^{\prime k}(\tau)\right. \\
& \left.-g_{i}\left(\tilde{x}_{1}^{k}(\tau), \ldots, \tilde{x}_{n}^{k}(\tau)\right)\right) \mathrm{d} \tau,
\end{aligned}
$$


we obtain stationary conditions $\left.\lambda_{i}^{\prime}(\tau)\right|_{\tau=t}=0$ and $1+$ $\left.\lambda_{i}(\tau)\right|_{\tau=t}=0, i=1,2, \ldots, n$.

Therefore, the Lagrange multipliers can be easily identified as

$$
\lambda_{i}=-1, i=1,2, \ldots, n .
$$

Substituting the Lagrange multipliers (9) into the correctional functional (8), we acquire the following SVIM formula

$$
\begin{aligned}
& x_{i}^{k+1}(t)=x_{i}^{k}(t) \\
& \quad-I_{\alpha}^{t^{*}}\left(T_{\alpha} x_{i}^{k}(\tau)-g_{i}\left(\tilde{x}_{1}^{k}(\tau), \ldots, \tilde{x}_{n}^{k}(\tau)\right)\right) \mathrm{d} \tau,
\end{aligned}
$$

for $i=1,2, \ldots, n$. If we begin with initial conditions $x_{i, 0}\left(t^{*}\right)=x_{i, 0}\left(t_{0}\right)=x_{i}(0)$, the iteration formula of the SVIM (10) can be carried out within every subinterval of equal length $\Delta t$, and so all solutions $x_{i}^{k}(t)$, $(i=1,2, \ldots, n ; k=1,2, \ldots)$ are completely determined.

\section{Numerical results}

In this section, we give two examples to illustrate the effectiveness and applicability of the proposed conformable fractional model (6). The SVIM is applied and compared with the VIM and fourth order Runge-Kutta method (RK4).

Example 1: Consider the following nonlinear programming problem [27, No. 216],

$$
\begin{array}{cl}
\text { minimize } & f(x)=100\left(x_{1}^{2}-x_{2}\right)^{2}+\left(x_{1}-1\right)^{2}, \\
\text { subject to } & h(x)=x_{1}\left(x_{1}-4\right)-2 x_{2}+12=0 .
\end{array}
$$

Firstly, the problem (11) is transformed to the unconstrained optimization problem with penalty function (2) for $\gamma=2$. Then we have

$$
\begin{gathered}
F(x, \eta)=100\left(x_{1}^{2}-x_{2}\right)^{2}+\left(x_{1}-1\right)^{2} \\
+\frac{1}{2} \eta\left(x_{1}\left(x_{1}-4\right)-2 x_{2}+12\right)^{2}
\end{gathered}
$$

where $\eta \in \mathbb{R}^{+}$is an auxiliary penalty variable. Thereafter, the corresponding conformable fractional gradient based dynamic system from (6) is defined as

$$
\begin{gathered}
T_{\alpha} x_{1}(t)=-400\left(x_{1}^{2}-x_{2}\right) x_{1}-2\left(x_{1}-1\right) \\
\quad-\eta\left(2 x_{1}-4\right)\left(x_{1}^{2}-4 x_{1}-2 x_{2}+12\right) \\
T_{\alpha} x_{2}(t)=200\left(x_{1}^{2}-x_{2}\right)+ \\
2 \eta\left(x_{1}^{2}-4 x_{1}-2 x_{2}+12\right) \\
x_{1}(0)=0, x_{2}(0)=0
\end{gathered}
$$

where $0<\alpha \leq 1$.

Finally, to obtain the solutions of (12) the SVIM and VIM are implemented with auxiliary penalty variable $\eta=800$, step size $\Delta T=0.00001$ and Lagrange multipliers $\lambda_{i}=-1$, using the following iteration formula,

$$
\begin{aligned}
& x_{i}^{k+1}(t)=x_{i}^{k}(t)-I_{\alpha}^{t^{*}}\left(T_{\alpha} x_{i}^{k}(\tau)\right. \\
& \left.\quad-g_{i}\left(\tilde{x}_{1}^{k}(\tau), \ldots, \tilde{x}_{n}^{k}(\tau)\right)\right) \mathrm{d} \tau, \quad i=1,2 .
\end{aligned}
$$

In Fig. 1, it can clearly be seen that the conformable fractional model reaches the optimal solutions of (11) after only one iteration using the SVIM. It can also be verified that SVIM obtains the optimal solutions faster than other methods.
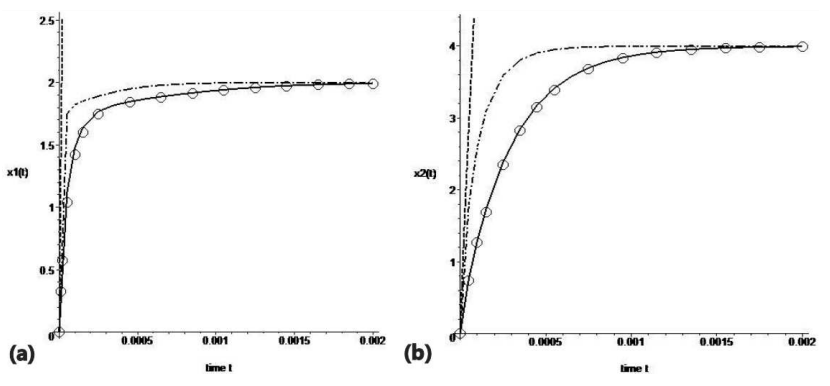

Fig. 1. Comparison of $x(t)$ for problem (11). Dash: $\operatorname{VIM}(\Delta T=0.00001)$ for $\alpha=0.9$, Dashdot: SVIM $(\Delta T=0.00001)$ for $\alpha=0.9$, Solidline: $\operatorname{SVIM}(\Delta T=$ $0.00001)$ for $\alpha=1$, Circles: RK4 $(\Delta T=0.00001)$ for $\alpha=1$.

Example 2: Consider the equality constrained problem [26, No. 76],

$$
\begin{gathered}
\operatorname{minimize} \quad f(x)=\left(x_{1}-1\right)^{2}+\left(x_{1}-x_{2}\right)^{2} \\
+\left(x_{2}-x_{3}\right)^{2}+\left(x_{3}-x_{4}\right)^{4} \\
+\left(x_{4}-x_{5}\right)^{4}
\end{gathered}
$$

subject to $h_{1}(x)=x_{1}+x_{2}^{2}+x_{3}^{3}-2-3 \sqrt{2}=0$,

$$
\begin{aligned}
& h_{2}(x)=x_{2}-x_{3}^{2}+x_{4}+2-2 \sqrt{2}=0, \\
& h_{3}(x)=x_{1} x_{5}-2=0 .
\end{aligned}
$$

This is a practical problem with an unknown exact solution. The conformable fractional gradient based system for (13) is obtained through the previous steps as follows:

$$
\left.\begin{array}{l}
T_{\alpha} x_{i}(t)=-\nabla_{x_{i}} f(x)-\eta \sum_{m=1}^{3} \nabla_{x_{i}} h_{m}(x), \\
x_{1}(0)=2, x_{2}(0)=2, x_{3}(0)=2, \\
x_{4}(0)=2, x_{5}(0)=2,
\end{array}\right\}
$$

where $0<\alpha \leq 1$ is the order of the conformable fractional derivative. Utilizing the SVIM (10) and VIM (5) with penalty variable $\eta=300$, step size $\Delta T=0.00001$ and Lagrange multipliers $\lambda_{i}=-1, i=1,2,3,4,5$; the solutions are acquired as shown in Tables I-III. In numerical simulation, the VIM solutions for $\alpha=0.9$ are divergent.

TABLE I

The value of $x(t)$ for problem (13) obtained from SVIM $(\alpha=0.9)$

\begin{tabular}{c|c|c|c|c|c}
\hline \hline \multicolumn{6}{c}{ SVIM $(\alpha=0.9)$} \\
\hline$t$ & $x_{1}(t)$ & $x_{2}(t)$ & $x_{3}(t)$ & $x_{4}(t)$ & $x_{5}(t)$ \\
\hline 0.000 & 2.00000 & 2.00000 & 2.00000 & 2.00000 & 2.00000 \\
1.000 & 1.19245 & 1.36356 & 1.47219 & 1.63214 & 1.67721 \\
2.000 & 1.19122 & 1.36267 & 1.47275 & 1.63475 & 1.67894 \\
3.000 & 1.19112 & 1.36259 & 1.47280 & 1.63492 & 1.67908 \\
4.000 & 1.19111 & 1.36258 & 1.47280 & 1.63494 & 1.67909 \\
5.000 & 1.19111 & 1.36258 & 1.47280 & 1.63494 & 1.67909 \\
6.000 & 1.19111 & 1.36258 & 1.47280 & 1.63494 & 1.67909
\end{tabular}


TABLE II

The value of $x(t)$ for problem (13) obtained from SVIM $(\alpha=1)$.

\begin{tabular}{c|c|c|c|c|c}
\hline \hline \multicolumn{6}{c}{ SVIM $(\alpha=1)$} \\
\hline$t$ & $x_{1}(t)$ & $x_{2}(t)$ & $x_{3}(t)$ & $x_{4}(t)$ & $x_{5}(t)$ \\
\hline 0.000 & 2.00000 & 2.00000 & 2.00000 & 2.00000 & 2.00000 \\
1.000 & 1.20227 & 1.34998 & 1.47634 & 1.65807 & 1.66342 \\
2.000 & 1.19133 & 1.35990 & 1.47389 & 1.64085 & 1.67878 \\
3.000 & 1.19053 & 1.36211 & 1.47325 & 1.63713 & 1.67992 \\
4.000 & 1.19073 & 1.36172 & 1.47301 & 1.63593 & 1.67963 \\
5.000 & 1.19091 & 1.36222 & 1.47290 & 1.63542 & 1.67937 \\
6.000 & 1.19101 & 1.36250 & 1.47285 & 1.63518 & 1.67923
\end{tabular}

TABLE III

The value of $x(t)$ for problem (13) obtained from RK4 $(\alpha=1)$.

\begin{tabular}{c|c|c|c|c|c}
\hline \hline \multicolumn{5}{c}{ RK4 $(\alpha=1)$} \\
\hline$t$ & $x_{1}(t)$ & $x_{2}(t)$ & $x_{3}(t)$ & $x_{4}(t)$ & $x_{5}(t)$ \\
\hline 0.000 & 2.00000 & 2.00000 & 2.00000 & 2.00000 & 2.00000 \\
1.000 & 1.20162 & 1.34946 & 1.47666 & 1.65951 & 1.66436 \\
2.000 & 1.19102 & 1.35966 & 1.47405 & 1.64157 & 1.67922 \\
3.000 & 1.19038 & 1.36161 & 1.47333 & 1.63751 & 1.68013 \\
4.000 & 1.19066 & 1.36216 & 1.47306 & 1.63613 & 1.67973 \\
5.000 & 1.19088 & 1.36239 & 1.47293 & 1.63554 & 1.67942 \\
6.000 & 1.19100 & 1.36249 & 1.47287 & 1.63525 & 1.67925
\end{tabular}

\section{Conclusions}

In this study, the conformable fractional gradient based dynamic system is modelled for solving a certain class of equality constrained optimization problems. To obtain the optimal solution, the steepest descent direction is integrated into the dynamic model. In order to obtain convergence, the step variational iteration method (SVIM) is implemented with conformable integral definitions. Numerical comparisons between the fourth-order RungeKutta method(RK4), the SVIM $(\alpha=1$ and $\alpha=0.9)$ and VIM $(\alpha=0.9)$ are made, and the results show that the conformable fractional gradient based system is more suitable and stable than the integer order system in obtaining the optimal solutions to Eq. (1). In addition, the new model gives a better approximation than the approach in [25]. Furthermore, we see that the SVIM, which is integrated with the definition of the conformable integral, is a reliable tool for solving fractional order differential equations.

\section{References}

[1] A.T. Özturan, Acta Phys. Pol. A 128, B-93 (2015).

[2] A. Recioui, Acta Phys. Pol. A 128, B-7 (2015).
[3] M.J. Pazdanowski, Acta Phys. Pol. A 128, B-213 (2015).

[4] A.T. Özturan, Acta Phys. Pol. A 130, 14 (2016).

[5] D.G. Luenberger, Y. Ye, Linear and Nonlinear Programming, 3rd ed., Springer, New York 2008.

[6] K.J. Arrow, L. Hurwicz, H. Uzawa, Studies in Linear and Non-Linear Programming, Stanford University Press, California 1958.

[7] A.V. Fiacco, G.P. Mccormick, Nonlinear Programming: Sequential Unconstrained Minimization Techniques, John Wiley, New York 1968.

[8] H. Yamashita, Math. Program. 18, 155 (1976).

[9] C.A. Botsaris, J. Math. Anal. Appl. 63, 177 (1978).

[10] A.A. Brown, M.C. Bartholomew-Biggs, J. Optim. Theory Appl. 62, 371 (1989).

[11] Y.G. Evtushenko, V.G. Zhadan, Opt. Meth. Software 3, 237 (1994).

[12] J. Schropp, I. Singer Numer. Funct. Anal. Optim. 21, 537 (2000).

[13] S. Wang, X.Q. Yang, K.L. Teo, Comput. Optim. Appl. 25, 251 (2003).

[14] L. Jin, L.-W. Zhang, X. Xiao, Appl. Math. Comput. 190, 1030 (2007).

[15] L. Jin, Appl. Math. Comput. 206, 186 (2008).

[16] V. Shikhman, O. Stein, J. Optim. Theory Appl. 140, 117 (2009).

[17] N. Özdemir, F. Evirgen, Bull. Malays. Math. Sci. Soc. 33, 79 (2010).

[18] I. Podlubny, Fractional Differential Equations, Academic Press, New York 1999.

[19] J.H. He, Commun. Nonlinear Sci. Numer. Simul. 2, 235 (1997).

[20] R.Y. Molliq, N.S.M. Noorani, R.R. Ahmad, A.K. Alomari, Sains Malaysiana 42, 347 (2013).

[21] R. Khalil, M. Al Horani, A. Yousef, M. Sababheh, J. Comput. Appl. Math. 264, 65 (2014).

[22] T. Abdeljawad, J. Comput. Appl. Math. 279, 57 (2015).

[23] F. Evirgen, N. Özdemir, J. Comput. Nonlinear Dyn. 6, 021003 (2011).

[24] F. Evirgen, N. Özdemir, A fractional order dynamical trajectory approach for optimization problem with HPM. Fractional Dynamics and Control, Eds. D. Baleanu, J.A.T. Machado, A.C.J. Luo, Springer 2012, p. 145 .

[25] F. Evirgen, Int. J. Optimization Control: Theor. Applicat. (IJOCTA) 6, 75 (2016).

[26] W. Hock, K. Schittkowski, Test Examples for Nonlinear Programming Codes, Springer-Verlag, Berlin 1981.

[27] K. Schittkowski, More Test Examples For Nonlinear Programming Codes, Springer, Berlin 1987. 